\title{
Models of inflation and their predictions
}

\author{
David H. Lyth \\ Department of Physics, \\ Lancaster University, \\ Lancaster LA1 4YB. U. K.
}

\begin{abstract}
Taking field theory seriously, inflation model-building is difficult but not impossible. The observed value of the spectral index of the adiabatic density perturbation is starting to discriminate between models, and may well pick out a unique one in the forseeable future.
\end{abstract}

I shall summarise the present status of inflation model-building, and its comparison with observation. This is already a substantial area of research, and with the advent of new observations in the next few years it will become a major industry. For an extensive review with references, see [1].

I focus on the simplest paradigm, which following the usual scientific practice should be tested to destruction before complications are entertained. There is a slowly-rolling, single-component inflaton field, which experiences Einstein gravity and drives the observable Universe into a spatially flat condition. The gaussian adiabatic density perturbation, generated by the vacuum fluctuation of the inflaton field $\phi$, is solely responsible for the origin of structure.

During inflation, the potential $V(\phi)$ of the inflaton field $\phi$ satisfies the flatness conditions

$$
\epsilon \ll 1, \quad|\eta| \ll 1,
$$

where $\epsilon \equiv \frac{1}{2} M_{\mathrm{P}}^{2}\left(V^{\prime} / V\right)^{2}$ and $\eta \equiv M_{\mathrm{P}}^{2} V^{\prime \prime} / V$. The inflaton field satisfies the slow-roll approximation $3 H \dot{\phi}=-V^{\prime}$ where $H$ is the Hubble parameter given by $3 H^{2}=$ $V / M_{\mathrm{P}}^{2}$. To work out the predictions one needs the number of $e$-folds $N$ between a given epoch and the end of slow-roll inflation (with the inflaton $\phi$ ). Its small change is defined by $d N \equiv-H d t(=-d \ln a)$, which with the slow-roll approximation leads to

$$
N(\phi)=\int_{\phi_{\text {end }}}^{\phi} M_{\mathrm{P}}^{-2} \frac{V}{V^{\prime}} d \phi .
$$

Here $\phi_{\text {end }}$ marks the end of slow-roll inflation, caused by the failure of the flatness conditions or by the destabilization of a non-inflaton field. Often, the integral is 
dominated by the other limit $\phi$ in which case the predictions are independent of $\phi_{\text {end }}$.

The vacuum fluctuation of the inflaton field generates a gaussian adiabatic primordial density perturbation, whose conventionally-defined spectrum is given by

$$
\delta_{H}^{2}(k)=\frac{1}{75 \pi^{2} M_{\mathrm{P}}^{6}} \frac{V^{3}}{V^{\prime 2}} .
$$

The right hand side is evaluated at the value $\phi(k)$ which corresponds to horizon exit $k=a H$. It satisfies $d \ln k=-d N(\phi)$ and therefore $\ln \left(k_{\text {end }} / k\right)=N(\phi)$, where $k_{\text {end }}$ is the scale leaving the horizon at the end of slow-roll inflation. With Eq. (2), this determines $\phi(k)$ provided that we know the value of $N(\phi)$ when some reference scale leaves the horizon. This scale is conveniently taken to be the central scale probed by $\mathrm{COBE}, k_{\mathrm{COBE}} \simeq 7.5 H_{0}$ where $H_{0}$ is the present Hubble parameter. Depending on the history of the Universe, one has

$$
N_{\mathrm{COBE}} \simeq 60-\ln \left(10^{16} \mathrm{GeV} / V^{1 / 4}\right)-\frac{1}{3} \ln \left(V^{1 / 4} / T_{\text {reh }}\right)-\Delta N,
$$

where $T_{\text {reh }}$ is the reheat temperature and $\Delta N>0$ allows for matter domination and thermal inflation between reheating and nucleosynthesis (and any continuation of inflation after the epoch $\left.\phi_{\text {end }}\right)$.

Differentiating Eq. (3) with the aid of Eq. (2), the spectral index is

$$
\frac{n(k)-1}{2} \equiv \frac{d \delta_{H}}{d \ln k}=\eta-3 \epsilon .
$$

If $n$ is constant then $\delta_{H}^{2} \propto k^{n-1}$.

Inflation also generates gravitational waves with primordial spectrum $\mathcal{P}_{\text {grav }}(k)=$ $\frac{2}{M_{\mathrm{P}}^{2}}\left(\frac{H}{2 \pi}\right)^{2}$. No gravitational wave signal is seen in the cmb anisotropy, which translates into a bound $\epsilon \lesssim 0.1$. The signal will probably never be seen unless $\epsilon \gtrsim 10^{-3}$.

At $k_{\mathrm{COBE}}$, the COBE observations give the accurate normalization (ignoring gravitational waves) $\delta_{H}=1.91 \times 10^{-5}$, which corresponds to

$$
V^{1 / 4} / \epsilon^{1 / 4}=.027 M_{\mathrm{P}}=6.7 \times 10^{16} \mathrm{GeV} .
$$

The present bound $\epsilon \lesssim 0.1$ on gravitational waves implies $V^{1 / 4} \lesssim 3.6 \times 10^{16} \mathrm{GeV}$. Gravitation waves will never be detectable if $V^{1 / 4} \lesssim 1 \times 10^{16} \mathrm{GeV}$, and most inflation models give a lower value when normalized to satisfy Eq. (6).

Over the range of cosmological scales, say $H_{0}<k<10^{4} H_{0}$, there is an observational bound on the scale-dependence of $\delta_{H}$. Until recently uncertainties in the cosmological parameters allowed only the weak result $|n-1| \lesssim 0.2$, but new data give a preliminary result $|n-1|<0.05$ [2]. After Planck flies we shall probably know $n(k)$ with an uncertainty of \pm 0.01 .

At the most primitive level, a model of inflation consists of a form for $V(\phi)$, plus a prescription for $\phi_{\text {end }}$ if the latter is not determined by $V$ as happens in some 
hybrid inflation models. From a field theory viewpoint, one expects $V(\phi)$ to be schematically of the following form ${ }^{1}$

$$
\begin{aligned}
V & =V_{0}+\frac{1}{2} m^{2} \phi^{2}+M \phi^{3}+\frac{1}{4} \lambda \phi^{4} \\
& +\left(\tilde{m}^{4}+2 g \phi^{2}+g^{2} \phi^{4}\right) \ln (g \phi / Q) \\
& +\sum_{d=5}^{\infty} \lambda_{d} M_{\mathrm{P}}^{4-d} \phi^{d} \\
& +\left[\Lambda^{4+\alpha} \phi^{-\alpha}-\tilde{\Lambda}^{4 \pm \beta} \phi^{\mp \beta}\right] .
\end{aligned}
$$

In the first line are renormalizable tree-level terms, with the origin is chosen so that $V^{\prime}=0$; the coefficients can have either sign. In the second line is the one-loop correction due to a particle with mass $\tilde{m}$ and coupling $g$, valid if $g \phi \gtrsim \tilde{m}$. (It is suppressed at smaller $\phi$.) The renormalization scale $Q$ should be fixed at a typical relevant value of $g \phi$ to minimize higher loop contributions. One sums over particles with a plus/minus sign for bosons/fermions, and unbroken global supersymmetry would make the total vanish. During inflation susy is broken, but the $\phi^{4}$ term still vanishes, and the $\phi^{2}$ term may vanish, but one expects no cancellations between the contributions to the the constant term. The third line contains the non-renormalizable terms which summarise unknown Planck scale physics; the coefficients $\lambda_{d}$ are generically of order 1 , but supersymmetry can make a finite number of them tiny. (By appealing to a continuous global symmetry it can make them all tiny, but no such symmetry comes out of string theory.) The fourth line contains a $\phi^{-\alpha}$ term that might come from dynamical symmetry breaking, and a $-\phi^{\mp \beta}$ term that might come from mutated hybrid inflation. These terms will be present only in exceptional cases, unlike the others which are generic.

One who presumes to use field theory ought to take this expression seriously, and when that is done the flatness conditions Eq. (1) turn out to be extraordinarily difficult to satisfy. The non-renormalizable terms are obviously dangerous. So are the loop corrections, especially in the context of hybrid inflation where some coupling has to be substantial. Less obviously, a generic supergravity theory gives a prediction of the form $M_{\mathrm{P}}^{2} V^{\prime \prime} / V=1+\cdots$, in which case there has to be some cancellation whose origin is at present obscure.

The simplest proposed model, usually called chaotic inflation, is a monomial $V \propto \phi^{p}$ with $p$ usually 2 or 4 . Inflation takes place at $\phi>\phi_{\text {end }} \sim p M_{\mathrm{P}}$, giving $n-1=-(2+p) /(2 N)$ and significant gravitational waves $(\epsilon=p /(5 N))$.

If non-renormalizable terms are there, they kill the above model. To live with them one needs $\phi \lesssim M_{\mathrm{P}}$ or $\phi \ll M_{\mathrm{P}}$. (The latter case is preferable, but one has to watch the loop correction which generates a term $V^{\prime \prime} \propto \phi^{-2}$.) Making the reasonable assumption that only one term of Eq. (7) is relevant, Eq. (1) then requires $V \simeq V_{0}$.

1) The form is more restrictive if $\phi$ is a pseudo-Goldstone boson, but that hypothesis has not so far lead to an attractive model of inflation. 
TABLE 1. Predictions for the spectral index $n(k)$, using $N=\ln \left(k_{\text {end }} / k\right)$ where $k_{\text {end }}=a H$ at the end of slow-roll inflation. Constants $c, q$ and $A$ are positive while $\sigma$ and $p$ can have either sign. In the first two cases one expects $|c| \sim 10^{-1}$ to $10^{-2}$, and in the second case, one expects $|\sigma| \gtrsim|c|$.

\begin{tabular}{|llll|}
\hline Comments & $V(\phi) / V_{0} \simeq 1$ & $\frac{1}{2}(n-1)$ & $\frac{1}{2} \frac{d n}{d \ln k}$ \\
\hline Mass term & $1 \pm \frac{1}{2} c \frac{\phi^{2}}{M_{\mathrm{P}}^{2}}$ & $\pm c$ & 0 \\
Softly broken susy & $1 \pm \frac{1}{2} c \frac{\phi^{2}}{M_{\mathrm{P}}^{2}} \ln \frac{\phi}{A}$ & $\pm c+\sigma e^{ \pm c N}$ & $\mp c \sigma e^{ \pm c N}$ \\
Spont. broken susy & $1+c \ln \frac{\phi}{A}$ & $-\frac{1}{2 N}$ & $-\frac{1}{2} \frac{1}{N^{2}}$ \\
$p>2$ or $-\infty<p<1$ & $1-c \phi^{p}$ & $-\left(\frac{p-1}{p-2}\right) \frac{1}{N}$ & $-\left(\frac{p-2}{p-1}\right)\left(\frac{n-1}{2}\right)^{2}$ \\
$p$ integer $\leq-1$ or $\geq 3$ & $1+c \phi^{p}$ & $\frac{p-1}{p-2} \frac{1}{N_{\max }-N}$ & $-\left(\frac{p-2}{p-1}\right)\left(\frac{n-1}{2}\right)^{2}$ \\
\hline
\end{tabular}

TABLE 2. Some predicted values.

\begin{tabular}{|cllll|}
\hline$V(\phi) / V_{0}$ & \multicolumn{2}{c}{$1-n$} & \multicolumn{2}{c|}{$-10^{3} d n / d \ln k$} \\
& $N=50$ & $N=20$ & $N=50$ & $N=20$ \\
\hline $1+c \ln (\phi / Q)$ & 0.02 & 0.05 & $(0.4)$ & 2.6 \\
$1-c \phi^{-2}$ & 0.03 & 0.075 & $(0.6)$ & 3.8 \\
$1-c \phi^{4}$ & 0.06 & 0.15 & $(1.2)$ & 5.4 \\
$1-c \phi^{3}$ & 0.08 & 0.20 & $(1.6)$ & 10.0 \\
\hline
\end{tabular}

Predictions for the spectral index are given in the Tables. An inflaton field with negligible interaction $\left(V=V_{0} \pm \frac{1}{2} m^{2} \phi^{2}\right)$ gives a constant $n-1$, which can be positive or negative and is typically not extremely small or interactions would be significant. One significant interaction term typically gives $n$ close to 1 , with weak scale-dependence.

A dramatic exception is the case [3] where a $\phi^{2} \ln \phi$ loop correction dominates the mass term, as shown in the second line of Table 1 . The correct COBE normalization Eq. (6) is obtained with a reasonable value $c \sim 10^{-1}$ to $10^{-2}$ of the coupling $c^{2}{ }^{2}$ Furthermore, such a coupling allows $n$ to pass through 1 on cosmologically interesting scales!

The observed value of $n(k)$ will become an increasingly powerful discriminator in the future. If one were to take it seriously, the preliminary result $|n-1|<0.05$ would already rule out the cubic self-interaction in Table 2 . It would also strongly constrain the parameters $c$ and $\sigma$ in the case just mentioned, perhaps demanding physically unreasonable values for them.

\section{REFERENCES}

1. D. H. Lyth and A. Riotto, hep-ph/9807278, to appear in Phys. Reports.

2) To calculate this normalization one has to take into account higher loop corrections by using a renormalization-group-improved potential, but the order of magnitude is unaffected. 
2. R. Bond, Pritzker Symposium, http://www-astro-theory.fnal.gov/Personal/psw/talks/bond/bond.03.gif.

3. E. D. Stewart, Phys. Lett. B391, 34 (1997); Phys. Rev. D56, 2019 (1997). 